\title{
Efficacy and Safety of Interferon-Alpha $2 b$ for Patients with Hepatic Epithelioid Hemangioendothelioma: Outcomes of a Case-Series Analysis
}

\section{Xiaolei Liu \\ Zihuan Zhang \\ Jia Huang \\ Haidong Tan \\ Zhiying Yang}

Department of Hepatobiliary Surgery, China-Japan Friendship Hospital, Beijing, People's Republic of China
Correspondence: Zhiying Yang Department of Hepatobiliary Surgery,

China-Japan Friendship Hospital, 2

Yinghua Dongjie, Hepingli, Beijing,

100029, People's Republic of China

Tel +861084205054

Email yangzhy@aliyun.com
Background: Hepatic epithelioid hemangioendothelioma (HEH) is a rare tumor type. No effective medicine or standard treatment for HEH has been established.

Patients and Methods: From March 2014 to April 2021, 62 patients with pathologically diagnosed HEH were observed regularly, and interferon-alpha $2 b$ (IFN-a 2b) was administered to patients with progressive disease or reoccurrence. Adverse events (AEs) were assessed and recorded, and a tumor assessment scan was performed every 3 months.

Results: A total of 42 patients with HEH received IFN-a $2 b$ treatment in this study. No severe (grade $\geq 3$ ) AEs were reported in the group overall. The most common treatmentrelated AEs in patients receiving IFN-a $2 \mathrm{~b}$ were fever $(50.0 \%)$ and fatigue $(21.4 \%)$. Partial response and complete response were achieved in 20 patients $(47.6 \%)$ and 2 patients $(4.8 \%)$, respectively, and the objective response rate was $52.4 \%$. Stable disease was observed in 12 patients $(28.6 \%)$, and the disease control rate was $81.0 \%$. Progressive disease was observed in 8 patients $(19.0 \%)$. The 1-, 3-, and 5-year progression-free survival rates were $81.0 \%$, $69.2 \%$, and $62.3 \%$, respectively. Only 1 patient died as a result of disease progression during the study. The 1-, 3-, and 5-year overall survival rates were $100 \%, 97.2 \%$, and $97.2 \%$, respectively.

Conclusion: IFN-a $2 b$ is a safe and effective treatment for patients with HEH. The encouraging results with IFN-a $2 b$ use make it a promising option for patients who have other types of epithelioid hemangioendothelioma; additional clinical trials are needed.

Keywords: liver, epithelioid hemangioendothelioma, interferon, sarcoma, chemotherapy

\section{Introduction}

Epithelioid hemangioendothelioma (EH) was initially described in 1982 by Weiss and Enzinger; ${ }^{1}$ it can involve both soft tissues and visceral organs, including the liver, lung, spleen, and heart. Hepatic EH (HEH) is an extremely rare tumor type (estimated incidence of less than 1 in 1,000,000 worldwide) with a clinical course intermediately between benign hemangioma and angiosarcoma. ${ }^{2}$ Surgical resection, liver transplantation, chemotherapy, and immunotherapy have all been implemented in patients with HEH. However, because of the scarcity of the disease and the difficulty of including patients, prospective clinical trials are very hard to conduct. Liver transplantation has reportedly achieved satisfying long-term survival for patients with $\mathrm{HEH}^{3-7}$ However, organ donation shortages limit the accessibility of liver transplantation for most patients with HEH unless they are in critical condition. Clinical studies of surgical resection, anti-angiogenesis drugs, 
chemotherapy, or transarterial embolization (TAE) have reported indeterminate results. ${ }^{8-11}$ At present, no effective medicine or standard treatment has been established yet for patients with $\mathrm{HEH}$.

Interferon-alpha $2 \mathrm{~b}$ (IFN-a $2 \mathrm{~b}$ ) as an immunotherapy has been used to treat hematological malignancies. ${ }^{12}$ IFNa therapy for EH has also been proposed for tumor reduction and metastasis prevention. ${ }^{9,13,14}$ Since 2014, our team has been investigating patients with $\mathrm{HEH}$, and 62 patients are under regular follow-up now. According to our previous retrospective study, IFN-a $2 b$ showed effective responses in patients with HEH. ${ }^{15}$ After we observed a clinically responsive outcome, we suggested IFN-a $2 b$ treatment to patients with HEH progression or reoccurrence. This study aimed to report the clinical outcomes after IFN-a $2 b$ treatment in patients with HEH.

\section{Patients and Methods}

From March 2014 to April 2021, 62 patients with histologically diagnosed HEH (53 patients diagnosed by liver biopsy and 9 patients, by surgery) were involved in the study and were observed regularly. Our previous retrospective study showed a responsive effect of IFN-a $2 b$ in patients with HEH. ${ }^{15}$ IFN-a $2 \mathrm{~b}$ was suggested to patients who experienced disease progressive disease (PD) or reoccurrence after previous treatment, for whom no other effective medicine was eligible. Patients who accepted the suggestion received 3 million units of IFN-a $2 b$ by subcutaneous injection once every other day. Tolerability and safety were assessed each month by interviewing patients and monitoring the blood cell counts as well as the liver, thyroid, and renal functions until month 6 and then every 3 months. A tumor assessment scan, including computed tomography (CT) and magnetic resonance imaging (MRI), was performed every 3 months, and the tumor status was assessed according to the Response Evaluation Criteria in Solid Tumors Committee (RECIST). ${ }^{16}$ Treatment was continued until disease progression occurred, unacceptable toxicity developed, or patients decided to terminate the medication. For patients who had HEH with extrahepatic metastases, the tumor assessment was based on the intrahepatic lesions. Safety assessments consisted of the monitoring and recording of adverse events (AEs) according to Common Terminology Criteria for Adverse Events version 4.03, laboratory evaluations, and vital signs. Patients with HEH who did not receive IFN-a $2 b$ treatment were observed regularly to record disease management and outcomes.
Informed consent forms were signed by all the patients who accepted the IFN-a $2 b$ treatment. The study protocol was approved by the ethics committee of the China-Japan Friendship Hospital, and the study was conducted in accordance with the principles of the Declaration of Helsinki and Good Clinical Practice Guidelines.

\section{Statistical Analysis}

Descriptive statistics were used to summarize patient characteristics. Progression-free survival (PFS) and overall survival (OS) were estimated using the Kaplan-Meier method. PFS was calculated from the start of IFN-a $2 b$ treatment to the first evidence of PD. OS was calculated from the start of IFN-a $2 b$ treatment to the time of death from any cause or last follow-up. Patients who remained alive or who were lost to follow-up had their data censored at the last contact. All statistical analyses were performed using SAS version 9.4 (SAS Institute, Cary, NC).

\section{Results \\ Patients}

Overall, a total of 62 patients with HEH were regularly observed in our clinic. Ten patients chose to maintain observation without treatment, and 6 patients without reoccurrence after surgery were observed regularly. Four patients chose targeted therapy (2 received apatinib; 1, sorafenib; and 1, sunitinib). Forty-two patients with HEH that progressed or recurred received IFN-a $2 b$ treatment, including 25 patients with tumor progression during observation, 11 patients with recurrence after curatively intended surgery or radiofrequency ablation, and 6 patients with tumor progression after chemotherapy or TAE (Table S1). The median age of patients who received IFN-a $2 b$ was 36 years (range, 18-65 years), and genders were almost equally distributed (52.4\% were men and $47.6 \%$ were women). The median time between diagnosis and initiation of IFN-a $2 b$ treatment was 9 months (range, 2-57 months).

At baseline, all 42 patients had an Eastern Cooperative Oncology Group performance status (ECOG PS) of 0 and no severe liver injury. Mild abdominal pain occurred in 9 patients, but no other symptom was recorded. Extrahepatic metastases were detected in 23 patients, and $50 \%$ patients had lung metastases (Table 1).

\section{Safety of IFN-a $2 b$}

No severe (grade $\geq 3$ ) AEs from IFN-a $2 b$ were recorded in the group. The most common treatment-related AEs were 
Table I Baseline Demographic and Clinical Characteristics of Patients with IFN-a $2 b(\mathrm{~N}=42)$

\begin{tabular}{|c|c|}
\hline Characteristics & No. (\%) \\
\hline \multicolumn{2}{|l|}{ Age, years } \\
\hline Median & 36 \\
\hline Range & $18-65$ \\
\hline \multicolumn{2}{|l|}{ Gender } \\
\hline Male & $22(52.4)$ \\
\hline Female & $20(47.6)$ \\
\hline \multicolumn{2}{|l|}{ Symptoms } \\
\hline Abdominal pain & $9(21.4 \%)$ \\
\hline None & $33(78.6 \%)$ \\
\hline \multicolumn{2}{|l|}{ Number of intrahepatic lesions } \\
\hline Multiple & $40(95.2)$ \\
\hline Solitary & $2(4.8)$ \\
\hline \multicolumn{2}{|l|}{ Involved disease sites } \\
\hline Lung & $21(50.0)$ \\
\hline Bone & $2(4.8)$ \\
\hline Spleen & I (2.4) \\
\hline Abdomen & I (2.4) \\
\hline None & $19(45.2)$ \\
\hline \multicolumn{2}{|l|}{ Inclusion reasons } \\
\hline Progression with no treatment & $25(59.5)$ \\
\hline Reoccurrence after surgery or RF & II (26.2) \\
\hline Failure of previous treatment & $6(14.3)$ \\
\hline \multicolumn{2}{|l|}{ ECOG PS } \\
\hline 0 & $42(100)$ \\
\hline I & $0(0)$ \\
\hline \multicolumn{2}{|l|}{ Range (median) serum liver function } \\
\hline$(A L T), U / L$ & 16-79 (24) \\
\hline (AST), U/L & $8-83(25)$ \\
\hline (ALP), U/L & $25-226(93)$ \\
\hline (GGT), U/L & $7-177(4 I)$ \\
\hline (Range of TB), $\mu \mathrm{mol} / \mathrm{L}$ & $9.4-44.8(12.7)$ \\
\hline
\end{tabular}

Abbreviations: ALP, alkaline phosphatase; ALT, alanine aminotransferase; AST, aspartate aminotransferase; ECOG PS, Eastern Cooperative Oncology Group performance status; GGT, gamma-glutamyl transpeptidase; IFN-a $2 b$, interferon-alpha $2 b$; RF, radiofrequency ablation; TB, total bilirubin.

fever $(50.0 \%)$ and fatigue $(21.4 \%)$. Fever occurred most often after the first several shots and disappeared within 1 month. Lasting fever was not recorded. Other AEs included leukopenia, thrombocytopenia, alopecia, rash, decreased appetite, anemia, and hypothyroidism (Table 2). Two patients had grade 2 hypothyroidism, and one patient had grade 2 leukopenia and thrombocytopenia. Thirteen patients $(31.0 \%)$ had no treatment-related AEs. No patients had treatment-related AEs that led to the interruption of IFN-a 2 b therapy.
Table 2 Treatment-Related Adverse Events with IFN-a 2b $(\mathrm{N}=42)$

\begin{tabular}{|l|c|c|c|}
\hline \multirow{2}{*}{ Adverse Events } & \multicolumn{3}{|c|}{ No. (\%) } \\
\cline { 2 - 4 } & Any Grade & Grade I & Grade 2 \\
\hline Fever & $2 \mathrm{I}(50.0)$ & $2 \mathrm{I}(50)$ & 0 \\
Fatigue & $9(21.4)$ & $9(2 \mathrm{I} .4)$ & 0 \\
Leukopenia & $2(4.8)$ & $\mathrm{I}(2.4)$ & $\mathrm{I}(2.4)$ \\
Thrombopenia & $2(4.8)$ & $\mathrm{I}(2.4)$ & $\mathrm{I}(2.4)$ \\
Rash & $2(4.8)$ & $2(4.8)$ & 0 \\
Alopecia & $2(4.8)$ & $2(4.8)$ & 0 \\
Hypothyroidism & $2(4.8)$ & 0 & $2(4.8)$ \\
Decreased appetite & $\mathrm{I}(2.4)$ & $\mathrm{I}(2.4)$ & 0 \\
Anemia & $\mathrm{I}(2.4)$ & $\mathrm{I}(2.4)$ & 0 \\
None & $\mathrm{I} 3(3 \mathrm{I} .0)$ & $\mathrm{I}$ & $\mathrm{I}$ \\
\hline
\end{tabular}

Abbreviation: IFN-a $2 b$, interferon-alpha $2 b$.

\section{Efficacy of IFN-a $2 b$}

The median follow-up time after IFN-a $2 b$ initiation was 33 months (range, 3-76 months). The median duration of IFN-a $2 b$ treatment was 19 months (range, 3-48 months). In the 42 IFN-treated patients, partial response (PR) and complete response (CR) were achieved in 20 patients $(47.6 \%)$ and 2 patients (4.8\%), respectively, and the objective response rate (ORR) was 52.4\% (Figures 1 and 2). The median time from the start of IFN-a $2 \mathrm{~b}$ to observation of tumor regression was 10 months (range, 3-22 months). Stable disease (SD) was observed in 12 patients $(28.6 \%)$, and the disease control rate (DCR) was $81.0 \%$. PD was observed in 8 patients $(19.0 \%)$ (Figure S1).

Of the 34 patients with disease control, treatment of IFN-a $2 b$ was terminated in 21 patients (PR, $n=14 ; C R$, $\mathrm{n}=2$; SD, $\mathrm{n}=5$ ), and all terminations except those in the 2 patients with $\mathrm{CR}$ were conducted in accordance with patient decisions. IFN-a $2 \mathrm{~b}$ treatment was continued in 13 patients ( $P R, n=6$; SD, $n=7$ ). Of the 8 patients with $\mathrm{PD}$, targeted therapy was used in 4 patients, and the other patients chose observation only.

PD was detected in 4 of the 21 patients who stopped IFN-a 2b treatment; PD occurred 10, 12, 12, and 36 months after the termination of IFN-a $2 b$ for each patient, respectively. The 1-, 3-, and 5-year PFS rates were $81.0 \%$, $69.2 \%$, and $62.3 \%$, respectively. Only one patient died as a result of disease progression during the study. The 1-, 3-, and 5-year OS rates were 100\%, 97.2\%, and 97.2\%, respectively. The flowchart and brief results of this study were depicted in Figure 3. Detailed outcomes information and radiological changes are provided in the Table S1 and Figure S1. 


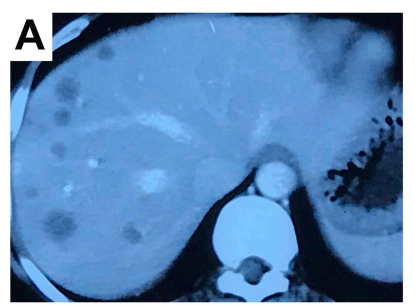

-6 months

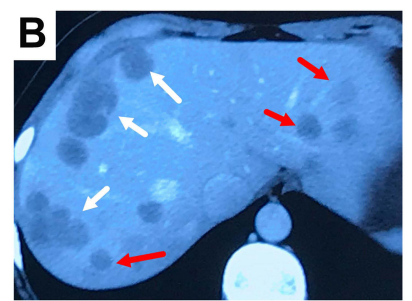

Baseline

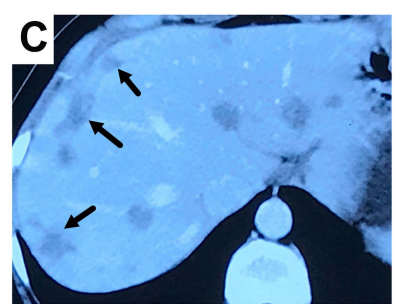

10 months

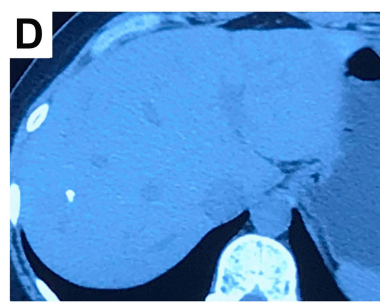

18 months

Figure I The complete response to interferon-alpha $2 \mathrm{~b}$ (IFN-a 2b) is illustrated in a female patient who had progressive disease after treatment with transarterial embolization (TAE). (A) Contrast-enhanced computed tomography (CT) scan reveals multiple intrahepatic lesions. (B) After TAE, intrahepatic tumors progressed (marked with white arrows), and new lesions occurred (marked with red arrows). Then, IFN-a $2 b$ treatment started. (C) Ten months after initiation of IFN-a $2 b$ treatment, the size of tumors had decreased significantly (marked with black arrows). (D) Eighteen months after initiation of IFN-a $2 b$ treatment, the intrahepatic tumors disappeared.

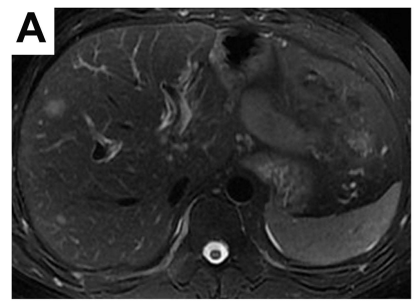

-24 months

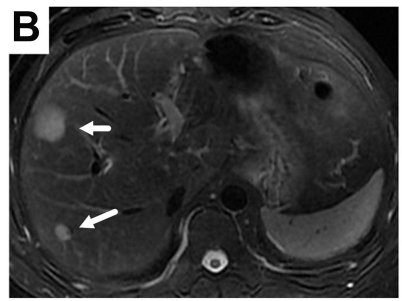

-12 months

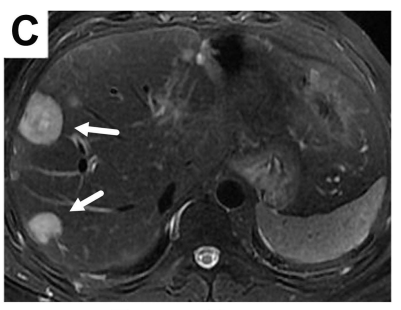

Baseline

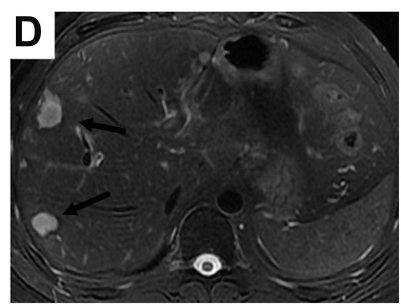

15 months

Figure 2 The partial response to interferon-alpha $2 b$ (IFN-a 2b) is illustrated in a male patient who experienced disease reoccurrence after surgery. (A) Small lesions were found 18 months after surgery. (B) Intrahepatic lesions (marked with white arrows) progressed after 12 months of observation. (C) Intrahepatic lesions (marked with white arrows) continued to progress after 24 months of observation, and IFN-a $2 b$ was treatment started. (D) Fifteen months after initiation of IFN-a $2 b$ treatment, the size of tumors decreased (marked with black arrows).

\section{Discussion}

$\mathrm{HEH}$ is an extremely rare hepatic tumor with varying biological behavior. Currently, there is no effective indicator to predict the malignancy. For most patients with $\mathrm{HEH}$, the tumor progresses slowly and no severe clinical symptoms manifest. Because of the rarity of the disease, patients with HEH are commonly misdiagnosed as having metastatic carcinoma. Although several studies have reported the radiological characteristics of $\mathrm{HEH},{ }^{17-20}$ a confirmed diagnosis still relies on histological examination.

No standard treatment has been established for HEH yet. Liver transplantation was reportedly effective, with acceptable long-term outcomes. ${ }^{4,7,21,22}$ However, the scarcity of organ donation limits the accessibility of liver transplantation for patients with HEH. Surgical resection is another treatment option, but the long-term results have been poor. ${ }^{23-25}$ Moreover, curative surgery is implausible for most patients with HEH because of the multicentricity of the tumor. Studies of chemotherapy, targeted therapy, or immunotherapy have reported indeterminate results. ${ }^{10,26-}$ 33 Thalidomide as anti-angiogenic therapy has reportedly controlled tumor progression, but a tumor response has seldom been achieved. ${ }^{8,34-37}$ Recently, a retrospective study of 38 patients with EH showed that sirolimus was an effective treatment, and PD was achieved in $75.7 \%$ of patients. ${ }^{38}$ Similar results have been reported using sirolimus in pediatric patients with EH. ${ }^{39}$ However, the molecular mechanism remains unclear.

IFN-a $2 \mathrm{~b}$ as an immunotherapy has been used to treat hematological malignancies. ${ }^{12}$ IFN-a therapy for $\mathrm{EH}$ has also been proposed for tumor reduction and metastasis prevention. ${ }^{9,13,14}$ Kayler et $\mathrm{al}^{40}$ reported that IFN- $\alpha$ was used to treat a metastatic HEH after liver transplantation. Antiproliferative activity of IFN-A, resulting from cancer cell growth inhibition, activation of immune cells, inhibition of vascularization, and induction of cytokines, has also been reported. ${ }^{12,41}$ Moreover, in our previous retrospective study, IFN-a $2 \mathrm{~b}$ achieved an effective response in 6 patients with HEH (PR, $n=4 ; C R, n=1 ; S D, n=1)$, which suggests that it is a promising treatment option. ${ }^{15}$ However, all the previous studies were case reports, and no long-term results of IFN-a $2 b$ treatment for a large group of patients with HEH have been reported.

Since 2014, 42 patients with progressive HEH have received IFN-a $2 b$ treatment in this study. PR and CR were observed in 22 patients, and the ORR was $52.4 \%$. SD was observed in 12 patients, and the DCR was $81.0 \%$. To our 


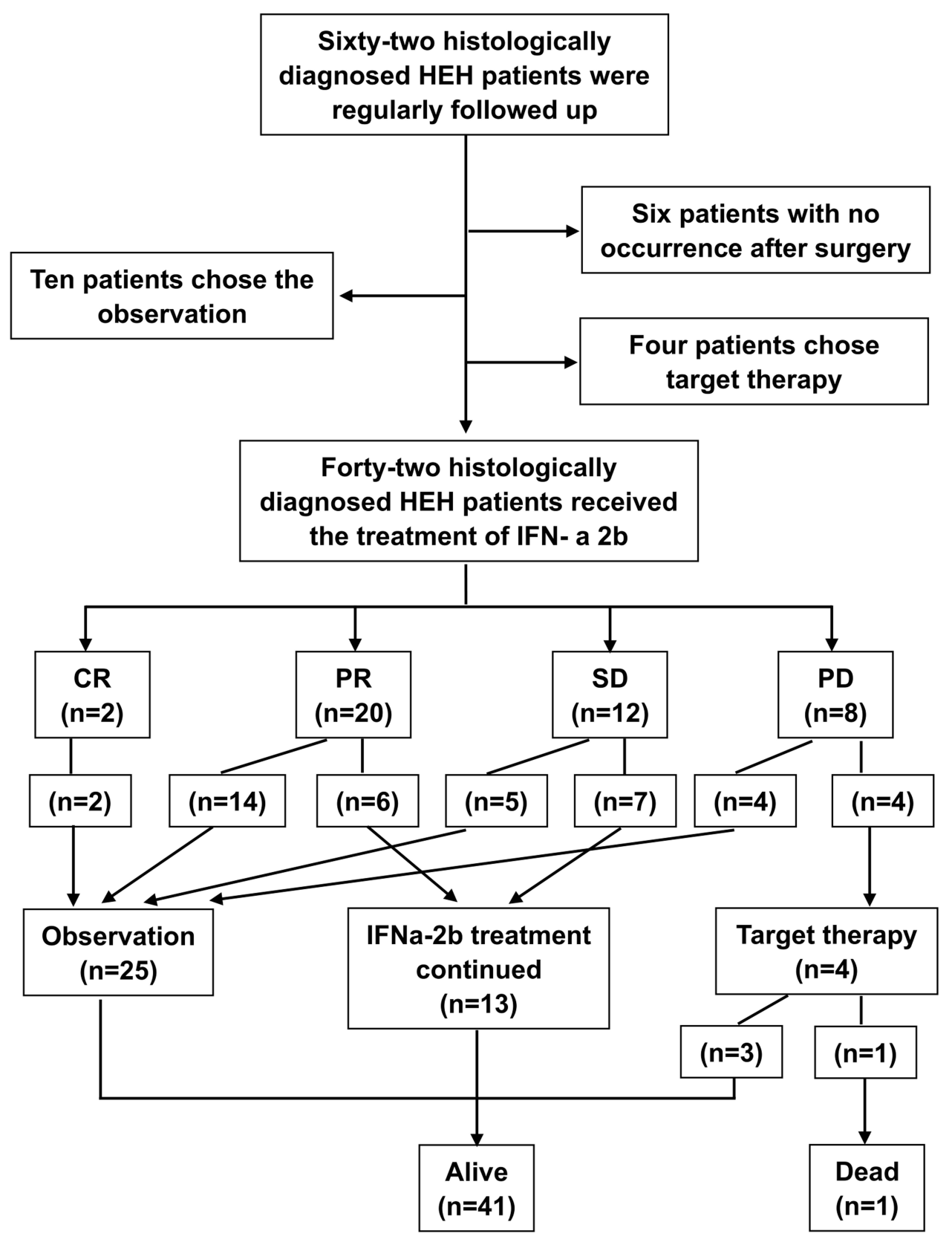

Figure 3 The flowchart and brief results of this study.

Abbreviations: $\mathrm{HEH}$, hepatic epithelioid hemangioendothelioma; $\mathrm{CR}$, complete response; PR, partial response; SD, stable disease; PD, progressive disease.

knowledge, this study indicates that IFN-a $2 \mathrm{~b}$ is the most effective medicine reported so far for patients with HEH. Moreover, compared with previous studies of liver transplantation that reported a 5-year survival rate of 70 $83 \%,{ }^{4,7}$ this study achieved a 5 -year survival rate of $97.2 \%$ in patients with HEH. Although the median followup time in this study was only 33 months, a tendency toward better long-term survival could be speculated. Moreover, the safety of long-term treatment with IFN-a $2 b$ was fully verified. The median duration of IFN-a $2 b$ therapy was 19 months, and no severe (grade $\geq 3$ ) AEs were recorded. Given the risk of hypothyroidism, the serum level of thyroxin should be monitored regularly in IFN-treated patients. The anti-tumor mechanism of IFN-a $2 b$ is not fully investigated or understood, but the encouraging results of this study provide evidence-based support for its use in HEH. Because EH also develops in other organs, such as lung and bone, additional clinical studies could be designed to verify the effect of IFN-a $2 b$ in other types of EH. Moreover, the combination of IFN-a $2 b$ and 
sirolimus may be a promising treatment option for patients with $\mathrm{HEH}$, and additional clinical studies to explore this option are needed.

In conclusion, IFN-a $2 b$ is a safe and effective treatment for patients with $\mathrm{HEH}$. Because of the extreme scarcity of the disease, a randomized clinical trial would be unrealistic. Although this was a single-arm study, the results still provided valuable clinical evidence for the treatment of HEH. The encouraging results observed with IFN-a $2 b$ treatment makes it a promising option for other types of $\mathrm{EH}$ as well and warrants additional clinical trial investigations.

\section{Data Sharing Statement}

All the detailed information and radiological images are provided in the Table S1 and Figure S1.

\section{Acknowledgments}

The authors thank all the associated staff of Radiology Department for assisting with the implementation of this study.

\section{Funding}

This study has no funding.

\section{Disclosure}

The authors declare that they have no conflicts of interest.

\section{References}

1. Weiss SW, Enzinger FM. Epithelioid hemangioendothelioma: a vascular tumor often mistaken for a carcinoma. Cancer. 1982;50 (5):970-981. doi:10.1002/1097-0142(19820901)50:5<970::AID-CNC R2820500527>3.0.CO;2-Z

2. Sardaro A, Bardoscia L, Petruzzelli MF, Portaluri M. Epithelioid hemangioendothelioma: an overview and update on a rare vascular tumor. Oncol Rev. 2014;8:259.

3. Lai Q, Feys E, Karam V, et al. Hepatic epithelioid hemangioendothelioma and adult liver transplantation: proposal for a prognostic score based on the analysis of the ELTR-ELITA registry. Transplantation. 2017;101(3):555-564. doi:10.1097/TP.0000000000001603

4. Rodriguez JA, Becker NS, O'Mahony CA, Goss JA, Aloia TA. Longterm outcomes following liver transplantation for hepatic hemangioendothelioma: the UNOS experience from 1987 to 2005. J Gastrointest Surg. 2008;12(1):110-116. doi:10.1007/s11605-007-0247-3

5. Nudo CG, Yoshida EM, Bain VG, et al. Liver transplantation for hepatic epithelioid hemangioendothelioma: the Canadian multicentre experience. Can J Gastroenterol. 2008;22(10):821-824. doi:10.1155/ 2008/418485

6. Nathan H, Pawlik TM. Limitations of claims and registry data in surgical oncology research. Ann Surg Oncol. 2008;15(2):415-423. doi:10.1245/s10434-007-9658-3

7. Lerut JP, Orlando G, Adam R, et al. The place of liver transplantation in the treatment of hepatic epithelioid hemangioendothelioma: report of the European liver transplant registry. Ann Surg. 2007;246 (6):949-957. doi:10.1097/SLA.0b013e31815c2a70
8. Mascarenhas RCV, Sanghvi AN, Friedlander L, Geyer SJ, Beasley HS, Van Thiel DH. Thalidomide inhibits the growth and progression of hepatic epithelioid hemangioendothelioma. Oncology. 2004;67(5-6):471-475. doi:10.1159/000082932

9. Galvao FHF, Bakonyi-Neto A, Machado MAC, et al. Interferon alpha-2B and liver resection to treat multifocal hepatic epithelioid hemangioendothelioma: a relevant approach to avoid liver transplantation. Transplant Proc. 2005;37(10):4354-4358. doi:10. 1016/j.transproceed.2005.11.022

10. Idilman R, Dokmeci A, Beyler AR, et al. Successful medical treatment of an epithelioid hemangioendothelioma of liver. Oncology. 1997;54(2):171-175. doi:10.1159/000227683

11. St Peter SD, Moss AA, Huettl EA, Leslie KO, Mulligan DC. Chemoembolization followed by orthotopic liver transplant for epithelioid hemangioendothelioma. Clin Transplant. 2003;17 (6):549-553. doi:10.1046/j.1399-0012.2003.00055.x

12. Zhang L, Tai YT, Ho MZG, Qiu L, Anderson KC. Interferon-alphabased immunotherapies in the treatment of B cell-derived hematologic neoplasms in today's treat-to-target era. Exp Hematol Oncol. 2017;6(1):20. doi:10.1186/s40164-017-0081-6

13. Calabrò L, Di Giacomo AM, Altomonte $\mathrm{M}$, et al. Primary hepatic epithelioid hemangioendothelioma progressively responsive to interferon-alpha: is there room for novel anti-angiogenetic treatments? J Exp Clin Cancer Res. 2007;26:145-150.

14. Radzikowska E, Szczepulska-Wójcik E, Chabowski M, Oniszh K, Langfort R, Roszkowski K. Pulmonary epithelioid haemangioendothelioma: interferon 2-alpha treatment-case report. Pneumonol Alergol Pol. 2008;76:281-285.

15. Liu XL, Yang ZY. Outcomes of hepatic epithelioid hemangioendothelioma with different managements: a retrospective investigation. Eur Rev Med Pharmacol Sci. 2021;25:4274-4282.

16. Therasse P, Arbuck SG, Eisenhauer EA, et al. New guidelines to evaluate the response to treatment in solid tumors. $J$ Natl Cancer Inst. 2000;92(3):205-216. doi:10.1093/jnci/92.3.205

17. Amin S, Chung H, Jha R. Hepatic epithelioid hemangioendothelioma: MR imaging findings. Abdom Imaging. 2011;36(4):407-414. doi:10.1007/s00261-010-9662-0

18. Azzam RI, Alshak NS, Pham HP. AIRP best cases in radiologic-pathologic correlation: hepatic epithelioid hemangioendothelioma. Radiographics. 2012;32(3):789-794. doi:10. 1148/rg.323115010

19. Chen Y, Yu RS, Qiu LL, Jiang DY, Tan YB, Fu YB. Contrastenhanced multiple phase imaging features in hepatic epithelioid hemangioendothelioma. World $J$ Gastroenterol. 2011;17 (30):3544-3553. doi:10.3748/wjg.v17.i30.3544

20. Lin J, Ji Y. CT and MRI diagnosis of hepatic epithelioid hemangioendothelioma. Hepatobiliary Pancreat Dis Int. 2010;9: 154-158.

21. Agrawal N, Parajuli S, Zhao P, et al. Liver transplantation in the management of hepatic epithelioid hemangioendothelioma: a single-center experience and review of the literature. Transplant Proc. 2011;43(7):2647-2650. doi:10.1016/j.transproceed.2011.06. 035

22. Fang C, Zhang W, Zheng S. Liver transplantation for hepatic epithelioid hemangioendothelioma: a case report and literature review. Int J Clin Exp Med. 2017;10:9693-9696.

23. Jung DH, Hwang S, Hong SM, et al. Clinicopathological features and prognosis of hepatic epithelioid hemangioendothelioma after liver resection and transplantation. Ann Transplant. 2016;21:784-790. doi:10.12659/AOT.901172

24. Penn I. Hepatic transplantation for primary and metastatic cancers of the liver. Surgery. 1991;110:726-734.

25. Lerut JP, Orlando G, Sempoux C, et al. Hepatic haemangioendothelioma in adults: excellent outcome following liver transplantation. Transpl Int. 2004;17(4):202-207. doi:10.1111/j.1432-2277.2004. tb00429.x 
26. Grotz TE, Nagorney D, Donohue J, et al. Hepatic epithelioid haemangioendothelioma: is transplantation the only treatment option? HPB. 2010;12(8):546-553. doi:10.1111/j.1477-2574.2010.00213.x

27. Soape MP, Verma R, Payne JD, Wachtel M, Hardwicke F, Cobos E. Treatment of hepatic epithelioid hemangioendothelioma: finding uses for thalidomide in a new era of medicine. Case Rep Gastrointest Med. 2015;2015:326795.

28. Chevreau C, Le Cesne A, Ray-Coquard I, et al. Sorafenib in patients with progressive epithelioid hemangioendothelioma: a Phase 2 study by the French Sarcoma Group (GSF/GETO). Cancer. 2013;119 (14):2639-2644. doi:10.1002/cncr.28109

29. Kobayashi N, Shimamura T, Tokuhisa M, Goto A, Ichikawa Y. Sorafenib monotherapy in a patient with unresectable hepatic epithelioid hemangioendothelioma. Case Rep Oncol. 2016;9(1):134-137. doi:10.1159/000443986

30. Sangro B, Iñarrairaegui M, Fernández-Ros N. Malignant epithelioid hemangioendothelioma of the liver successfully treated with sorafenib. Rare Tumors. 2012;4(2):e34. doi:10.4081/rt.2012.e34

31. Pinet C, Magnan A, Garbe L, Payan MJ, Vervloet D. Aggressive form of pleural epithelioid haemangioendothelioma: complete response after chemotherapy. Eur Respir J. 1999;14(1):237. doi:10.1034/j.1399-3003.1999.14a40.x

32. Woo JH, Kim TJ, Lee KS, Kim TS, Kim BT. Epithelioid hemangioendothelioma in the thorax: clinicopathologic, CT, PET, and prognostic features. Medicine. 2016;95(30):e4348. doi:10.1097/MD.00 00000000004348

33. Agulnik M, Yarber JL, Okuno SH, et al. An open-label, multicenter, Phase II study of bevacizumab for the treatment of angiosarcoma and epithelioid hemangioendotheliomas. Ann Oncol. 2013;24(1):25 7-263. doi:10.1093/annonc/mds237
34. Matthews SJ, McCoy C. Thalidomide: a review of approved and investigational uses. Clin Ther. 2003;25(2):342-395. doi:10.1016/ S0149-2918(03)80085-1

35. Kassam A, Mandel K. Metastatic hepatic epithelioid hemangioendothelioma in a teenage girl. J Pediatr Hematol Oncol. 2008;30 (7):550-552. doi:10.1097/MPH.0b013e31816e22d1

36. Raphael C, Hudson E, Williams L, Lester JF, Savage PM. Successful treatment of metastatic hepatic epithelioid hemangioendothelioma with thalidomide: a case report. J Med Case Rep. 2010;4(1):413. doi:10.1186/1752-1947-4-413

37. Salech F, Valderrama S, Nervi B, et al. Thalidomide for the treatment of metastatic hepatic epithelioid hemangioendothelioma: a case report with a long term follow-up. Ann Hepatol. 2011;10 (1):99-102. doi:10.1016/S1665-2681(19)31596-0

38. Stacchiotti S, Simeone N, Lo Vullo S, et al. Activity of sirolimus in patients with progressive epithelioid hemangioendothelioma: a case-series analysis within the Italian Rare Cancer Network. Cancer. 2021;127(4):569-576. doi:10.1002/cncr.33247

39. Engel ER, Cournoyer E, Adams DM, Stapleton S. A retrospective review of the use of sirolimus for pediatric patients with epithelioid hemangioendothelioma (EHE). J Pediatr Hematol Oncol. 2020;42 (8):e826-e829. doi:10.1097/MPH.0000000000001643

40. Kayler LK, Merion RM, Arenas JD, et al. Epithelioid hemangioendothelioma of the liver disseminated to the peritoneum treated with liver transplantation and interferon alpha-2B. Transplantation. 2002;74(1):128-130. doi:10.1097/00007890-200207150-00022

41. Asmana NR. Human interferon alpha-2b: a therapeutic protein for cancer treatment. Scientifica. 2014;2014:970315.
Cancer Management and Research

\section{Publish your work in this journal}

Cancer Management and Research is an international, peer-reviewed open access journal focusing on cancer research and the optimal use of preventative and integrated treatment interventions to achieve improved outcomes, enhanced survival and quality of life for the cancer patient.

\section{Dovepress}

The manuscript management system is completely online and includes a very quick and fair peer-review system, which is all easy to use. Visit http://www.dovepress.com/testimonials.php to read real quotes from published authors. 